Check for updates

Cite this: Phys. Chem. Chem. Phys., 2021, 23, 13250

Received 14th October 2020 Accepted 25th May 2021

DOI: $10.1039 / \mathrm{d} 0 \mathrm{cp} 05404 \mathrm{f}$

rsc.li/pccp

\title{
Low-frequency vibrational modes in G-quadruplexes reveal the mechanical properties of nucleic acids $\dagger$
}

\author{
Mario González-Jiménez, (D) Gopakumar Ramakrishnan, Nikita V. Tukachev, (D) \\ Hans M. Senn (iD and Klaas Wynne iD *
}

\begin{abstract}
Low-frequency vibrations play an essential role in biomolecular processes involving DNA such as gene expression, charge transfer, drug intercalation, and DNA-protein recognition. However, understanding the vibrational basis of these mechanisms relies on theoretical models due to the lack of experimental evidence. Here we present the low-frequency vibrational spectra of G-quadruplexes (structures formed by four strands of DNA) and B-DNA characterized using femtosecond optical Kerr-effect spectroscopy. Contrary to expectation, we found that G-quadruplexes show several strongly underdamped delocalized phonon-like modes that have the potential to contribute to the biology of the DNA at the atomic level. In addition, G-quadruplexes present modes at a higher frequency than B-DNA demonstrating that changes in the stiffness of the molecule alter its gigahertz to terahertz vibrational profile.
\end{abstract}

\section{Introduction}

The human genome contains in its transcriptional regulatory regions sequences rich in guanine that potentially can lose their double-helical structure in the presence of potassium cations to adopt a secondary conformation called a G-quadruplex (G4). ${ }^{1,2}$ This structure connects up to four strands of DNA (Fig. 1a) forming a stack of planar tetramers of guanine (G-quartets, Fig. 1b) linked together through Hoogsteen hydrogen bonds (Fig. 1c). ${ }^{3}$ The potassium cations, which fit into the central space, stabilize the structure by their interaction with the inner carbonyl oxygen atoms of the guanine bases. ${ }^{4}$

Although it is still a matter of debate whether G4 structures form in vivo, there is mounting evidence that they play an important role in the regulation of genes and oncogenes. ${ }^{5}$ Not only are G4 structures stable, but their prevalence is also controlled by the concentration of potassium ions, which is known to change with time and location within a cell. ${ }^{6}$ Furthermore, G4-promoting sequences are conserved through evolution $^{7}$ and there are a large number of proteins (Topo I, Pur1, MyoD, etc.) that have shown to have more affinity for G4s than for double-helix conformations in experiments in vitro ${ }^{8}$ and in vivo. ${ }^{9}$ Particularly important amongst these proteins is

School of Chemistry, University of Glasgow, Glasgow, G12 8QQ, UK.

E-mail: klaas.wynne@glasgow.ac.uk; Tel: +44 1413308522

$\dagger$ Electronic supplementary information (ESI) available: The data that support the findings of this study are available in Enlighten: Research Data Repository (University of Glasgow) with the identifier: http://dx.doi.org/10.5525/gla.researchdata.1150. See DOI: 10.1039/d0cp05404f the helicase protein encoded by the gene BLM, which is believed to undo G4s that are accidentally formed during recombination or replication. This gene is defective in Bloom's syndrome, a rare genetic disorder characterized by genomic instability and a very high incidence of cancer. ${ }^{10}$

G4 forming sequences are also abundant in the telomeres of chromosomes $^{1}$ and it has been suggested that these structures are formed between homologous chromosomes during meiosis. ${ }^{6}$ Furthermore, G4s inhibit the activity of telomerase-the enzyme that adds DNA to the ends of chromosomes to prevent them from shortening during cell division-that is often overexpressed in cancer cells. ${ }^{11}$

The need for a better understanding of the role of noncanonical structures in genetic function and the potential of G4s as therapeutic targets for anticancer drug development has triggered extensive research.

The attention of the physical sciences to these conformations has focused mainly on the characterization of structures, their thermodynamic stability and their rate of folding and unfolding. ${ }^{12-17}$ However, the dynamics of G4s have been almost completely ignored (except for some initial studies with NMR and 2DIR $)^{18}$ and their biological function is still unknown, despite the importance of low-frequency vibrations in the biomolecular processes in which G4s play a role. ${ }^{19}$

In this work, we present the first study of delocalized vibrational dynamics of G4s using femtosecond optical Kerr-effect (OKE) spectroscopy. OKE spectroscopy measures the low-frequency depolarized Raman spectrum in the time domain and is a technique that has proven to be valuable in the 

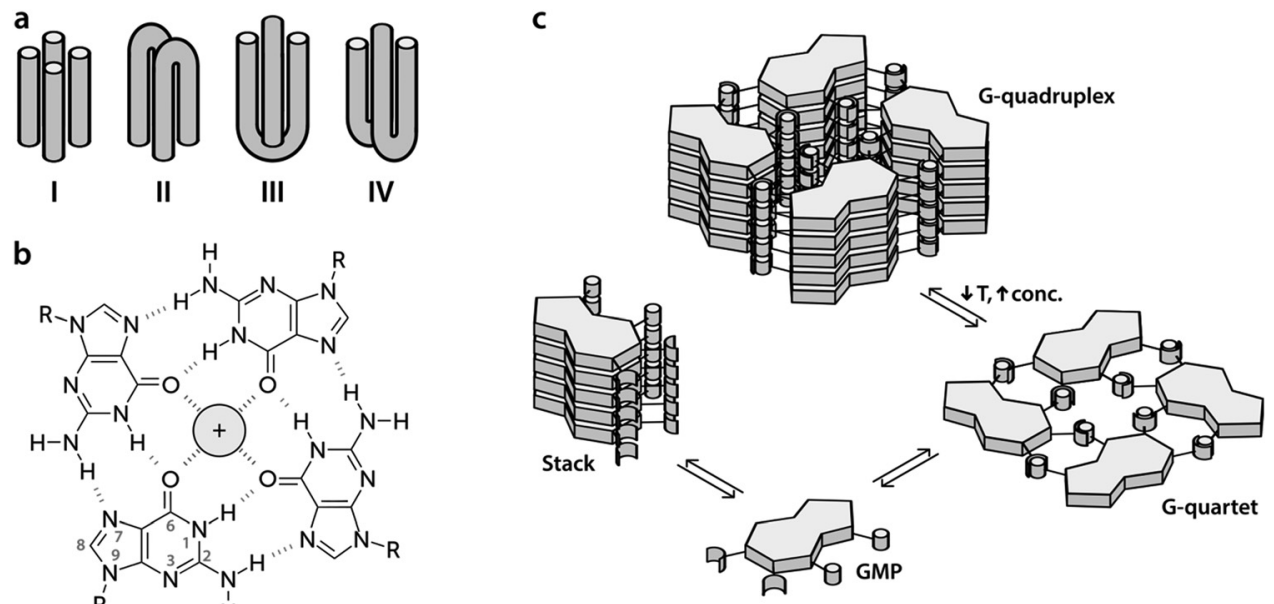

Fig. 1 Structure and topology of G-quartets and G-quadruplexes. (a) Different topological variants of G-quadruplexes (G4s) depending on the number of strands: I - tetramolecular G4, II - bimolecular and parallel dimer of hairpins, III - bimolecular and antiparallel dimer of hairpins, and IV intramolecular G4. (b) Chemical structure of a G-quartet coordinated with a metal cation. Blue dashes represent Hoogsteen hydrogen bonds and red dashes the metal-ligand bonding interaction between the monovalent cation and the inner oxygen (O6) atoms. (c) Equilibria involved in the selfassociation of guanosine monophosphate (GMP) in aqueous solution. The polar medium of the solvent promotes nucleotide stacking. Low temperatures and high concentrations contribute to the formation of G-quartets which are more stable in the G4 conformation.

investigation of the terahertz dynamics of $\mathrm{DNA}^{20,21}$ and other biomolecules in solution. ${ }^{22}$ We present the OKE spectra of $5^{\prime}$ guanosine monophosphate (GMP), a molecule that forms quartets and quadruplexes depending on the temperature and concentration (Fig. 1c). At low temperatures and high concentrations, the G4 conformation is favoured while at high temperatures and low concentrations, the equilibrium is shifted towards the formation of stacks. ${ }^{5}$ In order to disentangle the spectra of stacks, quartets, and G4s, we have compared the OKE spectra of GMP with the OKE spectra of solutions of the other DNA bases, adenosine, thymine, and cytosine monophosphate (AMP, TMP, and CMP), which cannot establish Hoogsteen bonds and just form stacks. ${ }^{23}$ We have also studied the effect of the size of the cations located at the central channel on the vibrational dynamics of G4s. While $\mathrm{Li}^{+}$is too small to effectively stabilize the structure, $\mathrm{Na}^{+}$is sized to be coordinated with four oxygen atoms in the G-quartet plane and $\mathrm{K}^{+}$is sandwiched between quartets where it is coordinated with oxygens from both planes. In some cases, depending on the sequence and topology, the $\mathrm{Na}^{+}$ion can also appear in the intermediate level between two quartets. ${ }^{24}$ Larger cations occupy the same position as potassium but distort the guanine quartets and stabilize the structure less. Finally, we have measured the OKE spectra of three DNA oligomers that form G4s with three different topological variants (see Fig. 1): a unimolecular G-quadruplex, a bimolecular complex formed from hairpin dimerization, and a parallel stranded tetraplex. We will show that these DNA conformations present a completely different vibrational dynamics from that of the single strands and double helix. This difference may be related to the biological function of the G4 structures, since the vibrational motions of the DNA influence the thermodynamics and mechanisms of the interaction between the nucleic acid and the proteins involved in genetic regulation.

\section{Methods}

\subsection{Materials}

Guanosine $5^{\prime}$-monophosphate disodium salt hydrate (from yeast, $\geq 99.9 \%$, Sigma), adenosine $5^{\prime}$-monophosphate disodium salt (99.7\%, Alfa Aesar), thymidine $5^{\prime}$-monophosphate disodium salt (99.7\%, Alfa Aesar), lithium chloride ( $\geq 99 \%$, Sigma), potassium chloride ( $\geq 99 \%$, Sigma), and caesium chloride ( $\geq 99 \%$, Sigma) were all used as received. Lyophilized, HSPF purified oligomers were prepared by Eurofins Genomics. HPLC gradient grade water from Fisher Chemical was used for preparing aqueous solutions. These solutions were filtered prior to the measurements using a $0.2 \mathrm{~mm}$ hydrophilic polytetrafluoroethylene (PTFE) filters (Millipore). DNA solutions were annealed by equilibrating them in a water bath at $363 \mathrm{~K}$, followed by slow cooling down to the desired temperature.

\subsection{OKE experimental details}

The OKE data were recorded in a standard time-domain step-scan pump-probe configuration and Fourier transformed to obtain the frequency-domain reduced depolarized Raman spectrum as described previously (19). A laser oscillator (Coherent Micra) provided $\sim 10 \mathrm{~nJ}$ pulses $(0.8 \mathrm{~W}$ average power) with a nominal wavelength of $800 \mathrm{~nm}$ at a repetition rate of $82 \mathrm{MHz}$ providing a $20 \mathrm{fs}$ pulse width in the sample. Time-domain OKE traces were acquired by scanning with a step size of $5 \mathrm{fs}$ up to a delay of $5 \mathrm{ps}$ and thereafter logarithmically increased to a maximum delay of $1.2 \mathrm{~ns}$. The sample was contained in a rectangular quartz cuvette (Starna, optical path: $1 \mathrm{~mm}$ ) held in a brass block that was temperature-controlled with a precision of $\pm 0.5 \mathrm{~K}$.

\subsection{NMR experimental details}

Proton NMR spectra were recorded in $\mathrm{D}_{2} \mathrm{O}$ with a Bruker AVIII $500 \mathrm{MHz}$ Spectrometer. 


\subsection{OKE data analysis}

The OKE spectra consist of several broad overlapping bands that are analysed through curve fitting to several analytical functions. At the lowest frequencies $(<300 \mathrm{GHz})$, one finds processes associated with diffusive orientational relaxation of the molecules. The diffusive orientational relaxation of the monomer aggregates and oligomers in solution has been fitted here with a Debye function

$$
S_{\mathrm{D}}(\omega)=\operatorname{Im} \frac{A_{\mathrm{D}}}{1+\mathrm{i} \omega \tau}
$$

where $A_{\mathrm{D}}$ is the amplitude of the band, $\omega$ is the angular frequency, and $\tau$ is the relaxation time. The band at a slightly higher frequency $(\sim 30 \mathrm{GHz})$, that can be assigned to the diffusive relaxation of water in the solvation shell of the solvents, cannot be fitted to a Debye function due to its much greater width. This band was modelled using a Cole-Cole function

$$
S_{\mathrm{CC}}(\omega)=\operatorname{Im} \frac{A_{\mathrm{CC}}}{1+(\mathrm{i} \omega \tau)^{\alpha}}
$$

where $A_{\mathrm{CC}}$ is the amplitude of the band and $\alpha$ is a parameter that accounts for the broadness of the observed band.

In the terahertz range, one finds bands from modes that are not diffusive but critically damped or underdamped. These originate in librations, vibrations, and phonon-like modes. These have been fitted using the Brownian oscillator model ${ }^{20}$

$$
S_{\mathrm{BO}}(\omega)=\operatorname{Im} \frac{A_{\mathrm{BO}} \omega_{0}^{2}}{\omega_{0}^{2}-\omega^{2}-2 \mathrm{i} \gamma \omega}
$$

where $\omega_{0}$ is the undamped oscillator angular frequency and $\gamma$ is the damping rate.

\subsection{Computational details}

All computations were performed using Gaussian 09. ${ }^{25}$ A PBE0 density functional with Grimme dispersion correction ${ }^{26}$ combined with a def2-SVP basis set was employed as the default method. The structural accuracy of the molecules was tested using M06-2X calculations employing a def2-TZVP basis set. Solvent effects were accounted for by using a polarizable continuum model (IEFPCM as implemented in Gaussian) with the dielectric constant $\varepsilon_{\text {static }}=78$ (water).

\section{Results}

\subsection{The OKE spectra of GMP}

The OKE spectra of disodium guanylate, $\mathrm{Na}_{2}\left(5^{\prime}\right.$-GMP), solutions were measured at temperatures between 283 and $358 \mathrm{~K}$ and concentrations between $0.1 \mathrm{M}$ and $1.18 \mathrm{M}$ (the solubility limit). To obtain the spectrum of solvated GMP, the contribution of bulk water was subtracted from the experimental OKE data (see Note S1 and Fig. S11-S13, ESI $\dagger$ ) and the resultant spectra are shown in Fig. 2 (a linear scale version of this figure is available in the ESI, $\dagger$ Fig. S1). The OKE spectrum of $\mathrm{NaCl}$ solutions of concentrations up to $4.0 \mathrm{M}$ were also measured and it was found that the contribution of the ions to the subtracted spectra is negligible (Fig. S2, ESI $\dagger$ ). The low-frequency part of all spectra is dominated by a band peaking at frequencies too low to be accessible in these experiments and that moves to a higher frequency as the temperature increases. This band is characteristic of diffusive orientational and translational relaxation of GMP and could be modelled by the combination of a Cole-Cole and a Debye function (see OKE Data Analysis).

The high-frequency part of the OKE spectra contained the most significant features with clear changes in shape as a function of temperature and concentration. At low temperature and high GMP concentration, the spectra show two clearly defined bands. The lower frequency band, peaking at $\sim 400 \mathrm{GHz}$, is unusually sharp for this frequency region. The higher frequency band in the region between approximately 1.5 and $4.5 \mathrm{THz}$ is structured and appears to be the combination of three separate peaks. There is a weak band with a maximum at $5.5 \mathrm{THz}$ that was sometimes difficult to observe due to the greater noise in the OKE spectra at high frequencies. ${ }^{22}$ On increasing the temperature or decreasing the GMP concentration, these sharp bands disappeared to leave the high-frequency region dominated by a wide band with a maximum at $1.6 \mathrm{THz}$ that at higher temperatures fused with the low-frequency diffusive-relaxation band.

It was found that the high-frequency part of all the spectra shown in Fig. 2 can be fitted accurately using a combination of two base spectra, each of these consisting of a number of Brownian oscillator functions (see Table S1, ESI $\dagger$ ). The first set, consisting of six Brownian oscillators (labelled B1-B6), fits the spectra of lower temperature and higher concentration. Fig. S3 (ESI $\dagger$ ) shows that a smaller number of Brownian oscillators fails to reproduce the characteristic spectral bumps and peaks of GMP solutions. The second set, consisting of two Brownian oscillators (labelled BA and BB), fits the opposite conditions of higher temperature and lower concentration. The relative amplitudes of the functions used in each set were nearly constant in all the spectra. It was found that the use of non-Lorentzian functions (i.e., Gaussians) did not allow a simpler, more accurate fit of all the 152 spectra shown in Fig. 2.

\subsection{OKE spectra of AMP and TMP}

Aqueous solutions of these other bases at concentrations from 0.1 to $1.6 \mathrm{M}$ were investigated to quantify the low-frequency vibrational modes associated with their structure (see Fig. 3; the OKE spectra for CMP were studied previously). ${ }^{20}$ The OKE spectra of the solutions of all three molecules could be accurately fitted with two Debye functions for the low-frequency diffusive part and two slightly under-damped Brownian oscillators for the high-frequency part. Thus, the spectra of the AMP, TMP, and CMP solutions have an identical structure (same number of functions and the same approximate frequencies, damping rates, and relative amplitudes) to that of GMP at high temperature and low concentration. This demonstrates that the bands labelled BA and BB in GMP correspond to vibrational modes associated with the stacking interaction of nucleotides.

\section{3. ${ }^{1} \mathrm{H}$ NMR spectra of GMP}

Since the temperature- and concentration-dependent OKE spectra of GMP can be fitted with two base spectra, and since 


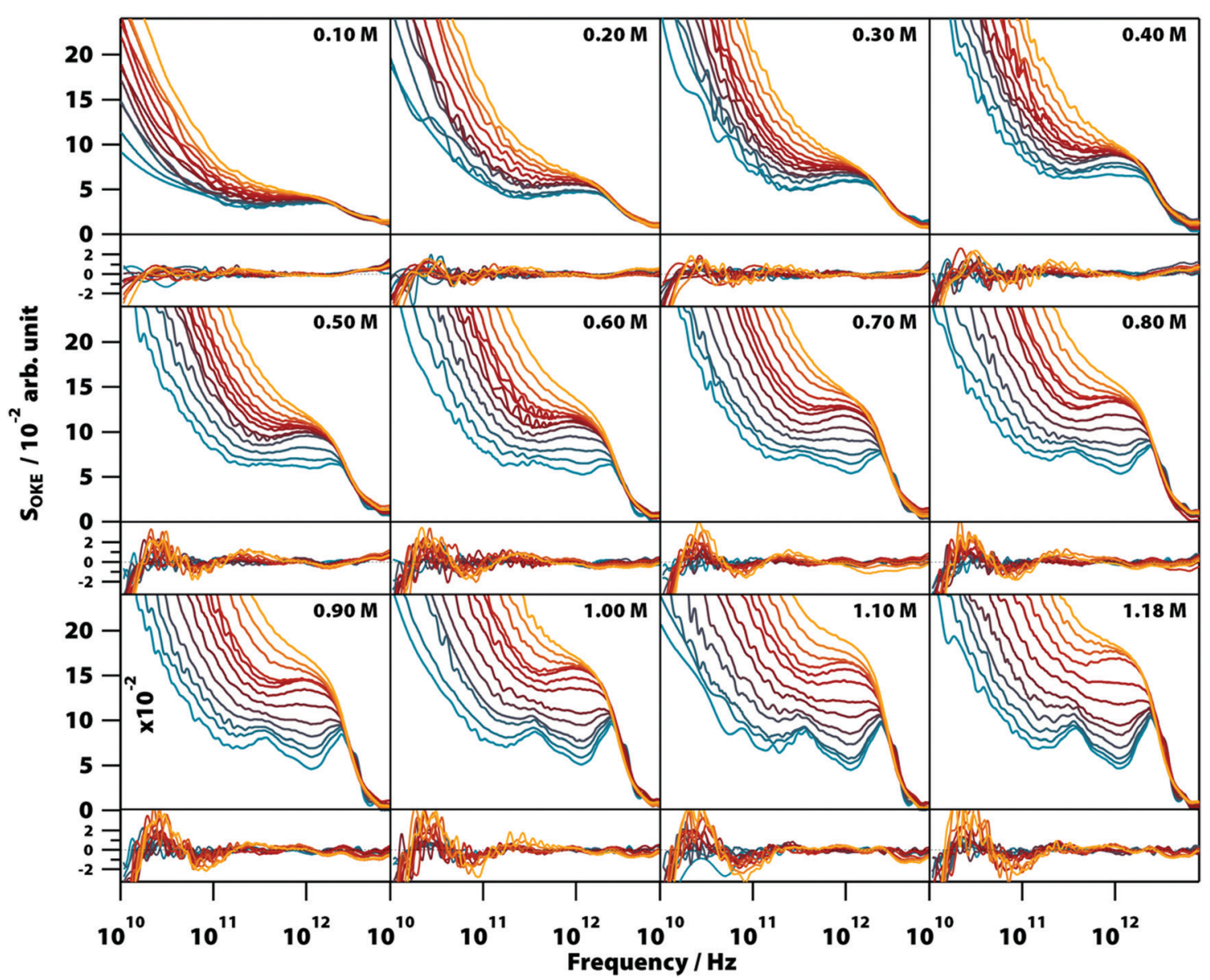

Fig. 2 Optical Kerr-effect spectra of aqueous $\mathrm{Na}_{2}\left(5^{\prime}\right.$-GMP) solutions at different concentrations and temperatures showing changes in the spectra due to the formation of stacks and G-quadruplexes. Temperatures run from 283 (blue) to $328 \mathrm{~K}$ (red) in steps of $5 \mathrm{~K}$ and from $328 \mathrm{~K}$ to $358 \mathrm{~K}$ (orange) in steps of $10 \mathrm{~K}$. The fitting deviations (residuals) between each spectrum and the proposed model values are shown in the graphs below the spectra.

one of these base spectra has now been assigned to stacked GMP, it implies that the other base spectrum corresponds to the G4 structure (see Fig. 1). This premise was confirmed by measuring the ${ }^{1} \mathrm{H}$ NMR spectra of a $1.18 \mathrm{M} \mathrm{Na}_{2}\left(5^{\prime}\right.$-GMP) solution between 283 and $353 \mathrm{~K}$ in intervals of $10 \mathrm{~K}$ (Fig. S5, ESI $\dagger$ ). At higher temperatures, these spectra show a sharp line around $8 \mathrm{ppm}$ corresponding to the hydrogen 8 (see Fig. 1) of the guanine ring of unassociated GMP. ${ }^{4}$ As the temperature dropped, the intensity of this peak decreases while four peaks attributed to the G4 structure appear between 6.2 and $8.2 \mathrm{ppm}^{17,27}$

\subsection{GMP equilibria and thermodynamics}

Averaging the intensity of the sets of bands in each base spectrum used to model the OKE data allowed us to determine precisely the proportion of the two conformations at each temperature and concentration (Fig. 4). From the dependence on temperature of the signal strength of the G4 structures, it is possible to determine their melting or denaturation temperatures for each initial concentration of GMP (Fig. S6 and Table S2, ESI $†$ ). The consistency of our calculated values with the NMR spectra and with the melting temperatures found in the literature ${ }^{17,28}$ also support our assignment of each base spectrum. Employing these melting temperatures, we could calculate a standard-state van't
Hoff enthalpy and entropy changes of $\Delta H^{\circ}=73 \pm 6 \mathrm{~kJ} \mathrm{~mol}^{-1}$ and $\Delta S^{\circ}=180 \pm 20 \mathrm{~J} \mathrm{~K}^{-1} \mathrm{~mol}^{-1}$ for the dissociation of the G-quadruplexes (Fig. S7 and Note S2, ESI $\dagger$ ).

\subsection{Influence of cations}

To gain more insight into the vibrational modes detected in the G4 structure, we compared in a broad range of temperatures (283-358 K) the OKE spectra of GMP. $\mathrm{Na}^{+}$with the OKE spectra of three GMP solutions prepared with an excess concentration of a different monovalent cation: lithium, potassium, and caesium. These cations replace sodium in the inner channel of the structure, altering its structure, stability, and dynamics due to their size difference. ${ }^{29}$ As a result, the solubility of GMP also changes, forcing the concentration of the measured solutions to be different. The spectra obtained are shown in the first row of Fig. 6 (a linear scale version can be found in Fig. S8, ESI $\dagger$ ) and could be modelled over the entire temperature span using the same number of Brownian oscillator functions employed in GMP. $\mathrm{Na}^{+}$solutions.

Moreover, the fit parameters for the spectra at higher temperatures (the functions labelled BA and BB in Fig. 6, third row) were equal, supporting the assignment to vibrations in a stack of nucleotides, since the cations of the medium do not 


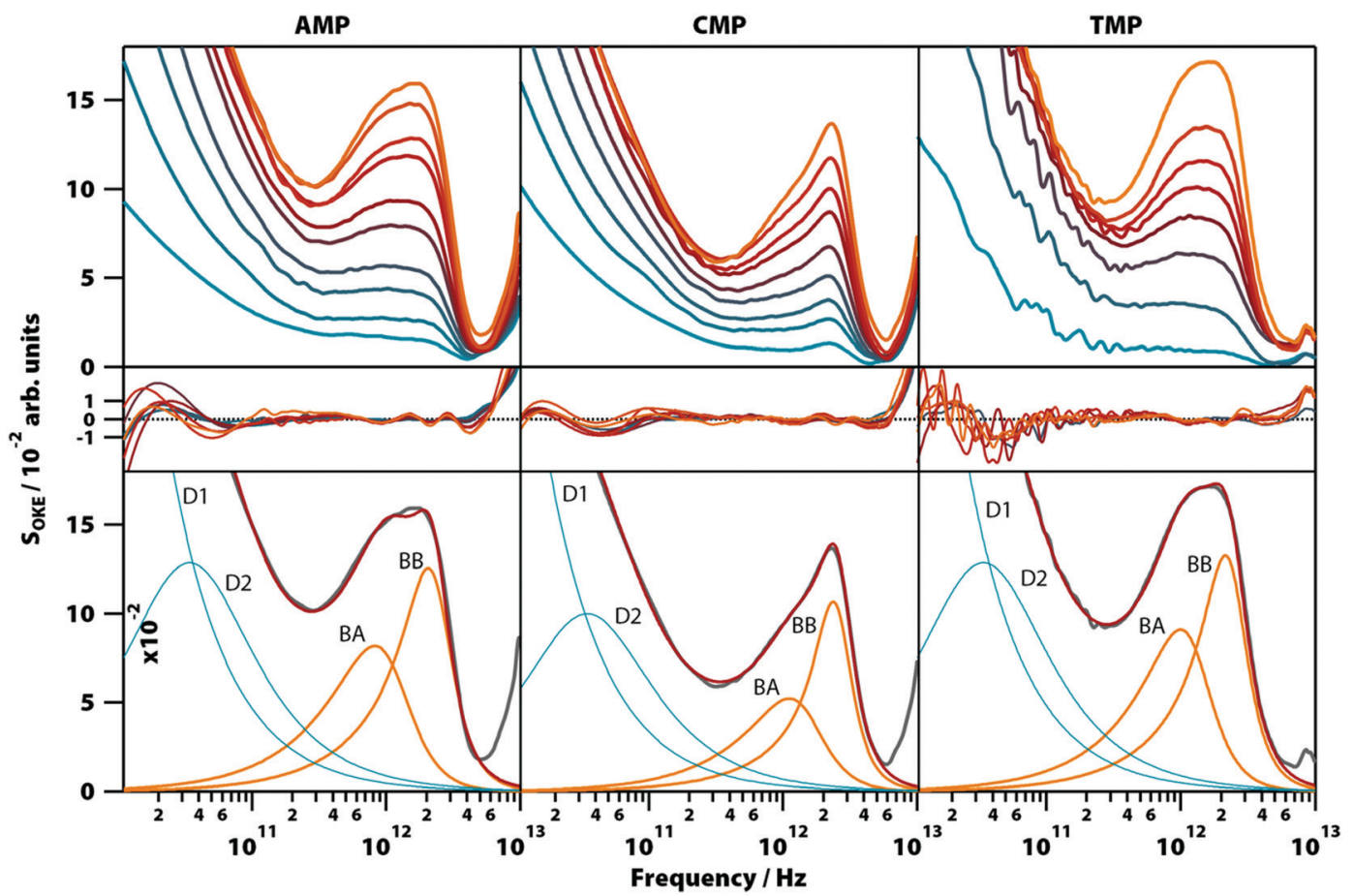

Fig. 3 OKE spectra of aqueous solutions of three non-GMP nucleotides at $298 \mathrm{~K}$ demonstrating the fundamentally different form. Shown are the spectra for adenine (left column), cytosine (central), and thymidine monophosphate (right) at concentrations between 0.1 (blue) and $1.6 \mathrm{M}$ (orange). Spectra are plotted in intervals of $0.1 \mathrm{M}$ between 0.1 and $0.4 \mathrm{M}$ and intervals of $0.2 \mathrm{M}$ between 0.4 and $1.6 \mathrm{M}$ for $\mathrm{AMP}$ and $\mathrm{CMP}$ and in intervals of $0.2 \mathrm{M}$ for the whole concentration range of TMP (first row). The second row shows the fitting deviations (residuals) between each spectrum and the fittings and the third row contains the 1.6 M spectrum for each nucleotide (grey), its fitting (red), and the functions used to model the data: Debye (blue) and Brownian oscillator (orange). A linear scale version of this figure is available in ESI $\dagger$ (Fig. S4).

take part in these structures. At low temperatures, the cation does influence the OKE spectra associated with G4s. In lithium and caesium solutions it is observed that the spectra, even at the low temperature of $283 \mathrm{~K}$, consist of the combination of the base spectra of both G4 and stacked GMP. This agrees with the fact that these cations are the ones that stabilize the structure the least; lithium because it is small in comparison with the central space and caesium because its size disrupts the structure. The characteristics of the bands are also affected by the size of the central cation. The bands of cations that are smaller than the central space (approximate radius: $131 \mathrm{pm}$, PDB entry: 1K8P), lithium (effective radius: $60 \mathrm{pm}$ ) and sodium $(95 \mathrm{pm}),{ }^{30}$ have essentially the same oscillatory parameters (Fig. 6, second row, and Table S1, ESI $\dagger$ ). However, the potassium cation, which is practically the size of the central space (radius: $133 \mathrm{pm}$ ), presents a spectrum very similar to that of sodium and lithium, except that its B4 band is displaced to a higher frequency position. The base spectrum when caesium is present (radius: $169 \mathrm{pm}$ ) has B1, B2 and B5 bands shifted to lower frequency positions, compared to the sodium spectrum.

\subsection{OKE spectra of oligonucleotide G4s}

In order to confirm that the narrow spectral band shapes seen in G4s constructed from GMP nucleotides are more general, aqueous solutions of three guanine-rich, G4-forming DNA oligomers were studied: thrombin-binding aptamer d(GGTTGGTGTGGTTGG) (TBA), which forms a unimolecular
G-quadruplex, $\mathrm{d}\left(\mathrm{G}_{3} \mathrm{CT}_{4} \mathrm{G}_{3} \mathrm{C}\right)$, which forms a dimer of two parallel hairpins, and d(TGGGGT), which forms a parallel-stranded tetraplex. The spectra from these oligomers are compared with that of a double-helix forming oligomer, $d\left(T_{2} \mathrm{AT}_{2} \mathrm{~A}_{3} \mathrm{TATAT}_{3} \mathrm{~A}_{2} \mathrm{TA}_{2}\right)$, studied previously in the conformation of B-DNA. ${ }^{20}$

Unlike with GMP, $\mathrm{Na}^{+}$does not sufficiently stabilize the G4 structures in guanine-rich oligomers and the presence of $\mathrm{K}^{+}$is required to form them (Fig. S9, ESI $\dagger$ ), so the solutions of the oligomers were prepared with a concentration of $1.65 \mathrm{M}$ of KCl (except TBA which formed a gel with that concentration and required $\mathrm{KCl} 0.4 \mathrm{M}$ ). The temperature-dependent OKE spectra (283 to $358 \mathrm{~K}$ ) are shown in Fig. 7 (a linear scale version of this figure is available in Fig. S10, ESI $\dagger$ ). As with GMP, the highfrequency part of these spectra can be fitted with two base spectra associated with a G4 structure and its denatured conformation.

The base spectrum of the G4 conformation is again observed at the lowest temperatures and, for the three oligomers, it can be adjusted with the same set of functions as used for the GMP solutions. Furthermore, the parameters of these Brownian oscillators (Table S1, ESI $\dagger$ ) deviate likewise from the models from G4 structures in GMP, suggesting that these vibrational modes have characteristics that appear only when nucleotides are bonded in oligomers. A comparison of these results with the model used to fit the double-helix spectrum ${ }^{20}$ (Fig. 7, right column) showed that bands B3 and B4 coincide at the same frequency with the bands BE and $\mathrm{BF}$ of the double-helix model, respectively. 


\begin{tabular}{|l|l|l|l|l|l|l|l|l|l|l|l|l|}
\hline T/K. $/ \mathrm{M}$ & $\mathbf{0 . 1 0}$ & $\mathbf{0 . 2 0}$ & $\mathbf{0 . 3 0}$ & $\mathbf{0 . 4 0}$ & $\mathbf{0 . 5 0}$ & $\mathbf{0 . 6 0}$ & $\mathbf{0 . 7 0}$ & $\mathbf{0 . 8 0}$ & $\mathbf{0 . 9 0}$ & $\mathbf{1 . 0 0}$ & $\mathbf{1 . 1 0}$ & $\mathbf{1 . 1 8}$ \\
\hline $\mathbf{2 8 3}$ & 0.00 & 0.00 & 0.65 & 3.53 & 7.08 & 8.58 & 13.03 & 17.14 & 19.26 & 25.07 & 26.02 & 27.54 \\
\hline $\mathbf{2 8 8}$ & 0.00 & 0.00 & 0.22 & 1.95 & 3.13 & 5.40 & 10.74 & 14.93 & 17.23 & 23.28 & 22.66 & 25.04 \\
\hline $\mathbf{2 9 3}$ & 0.00 & 0.00 & 0.00 & 0.41 & 1.53 & 2.94 & 8.35 & 12.40 & 15.50 & 20.59 & 20.87 & 23.76 \\
\hline $\mathbf{2 9 8}$ & 0.00 & 0.00 & 0.00 & 0.00 & 0.81 & 0.82 & 3.76 & 9.91 & 12.01 & 18.81 & 19.18 & 21.59 \\
\hline $\mathbf{3 0 3}$ & 0.00 & 0.00 & 0.00 & 0.00 & 0.42 & 0.53 & 1.07 & 5.39 & 8.89 & 14.78 & 17.08 & 17.51 \\
\hline $\mathbf{3 0 8}$ & 0.00 & 0.00 & 0.00 & 0.00 & 0.00 & 0.00 & 0.00 & 1.89 & 3.86 & 10.76 & 12.02 & 14.77 \\
\hline $\mathbf{3 1 3}$ & 0.00 & 0.00 & 0.00 & 0.00 & 0.00 & 0.00 & 0.00 & 0.51 & 1.20 & 4.31 & 8.73 & 9.18 \\
\hline $\mathbf{3 1 8}$ & 0.00 & 0.00 & 0.00 & 0.00 & 0.00 & 0.00 & 0.00 & 0.00 & 0.00 & 1.64 & 3.55 & 5.59 \\
\hline $\mathbf{3 2 3}$ & 0.00 & 0.00 & 0.00 & & 0.00 & 0.00 & 0.00 & 0.00 & & 0.21 & 1.09 & \\
\hline $\mathbf{3 2 8}$ & 0.00 & 0.00 & 0.00 & 0.00 & 0.00 & 0.00 & 0.00 & 0.00 & 0.00 & 0.00 & 0.00 & 2.76 \\
\hline $\mathbf{3 3 3}$ & 0.00 & 0.00 & 0.00 & 0.00 & 0.00 & 0.00 & 0.00 & 0.00 & 0.00 & 0.00 & 0.00 & 1.21 \\
\hline $\mathbf{3 3 8}$ & 0.00 & 0.00 & 0.00 & 0.00 & 0.00 & 0.00 & 0.00 & 0.00 & 0.00 & 0.00 & 0.00 & 0.00 \\
\hline $\mathbf{3 4 3}$ & 0.00 & 0.00 & 0.00 & 0.00 & 0.00 & 0.00 & 0.00 & 0.00 & 0.00 & 0.00 & 0.00 & 0.00 \\
\hline
\end{tabular}

\begin{tabular}{|l|l|l|l|l|l|l|l|l|l|l|l|l|}
\hline $\mathbf{r}$ c./M & $\mathbf{0 . 1 0}$ & $\mathbf{0 . 2 0}$ & $\mathbf{0 . 3 0}$ & $\mathbf{0 . 4 0}$ & $\mathbf{0 . 5 0}$ & $\mathbf{0 . 6 0}$ & $\mathbf{0 . 7 0}$ & $\mathbf{0 . 8 0}$ & $\mathbf{0 . 9 0}$ & $\mathbf{1 . 0 0}$ & $\mathbf{1 . 1 0}$ & $\mathbf{1 . 1 8}$ \\
\hline $\mathbf{2 8 3}$ & 8.39 & 10.98 & 13.84 & 20.94 & 15.98 & 12.77 & 11.52 & 9.33 & 5.53 & 2.88 & 0.43 & 0.58 \\
\hline $\mathbf{2 8 8}$ & 8.39 & 10.98 & 13.84 & 25.07 & 19.90 & 17.98 & 16.04 & 12.94 & 10.61 & 6.21 & 5.97 & 4.95 \\
\hline $\mathbf{2 9 3}$ & 8.39 & 10.98 & 13.99 & 25.07 & 25.49 & 22.84 & 18.56 & 17.76 & 13.87 & 10.58 & 8.05 & 6.46 \\
\hline $\mathbf{2 9 8}$ & 7.11 & 14.27 & 13.99 & 26.53 & 29.38 & 29.85 & 25.77 & 20.93 & 18.98 & 13.88 & 12.52 & 11.98 \\
\hline $\mathbf{3 0 3}$ & 7.11 & 16.05 & 13.99 & 26.53 & 29.38 & 32.78 & 30.99 & 27.97 & 24.07 & 19.97 & 15.81 & 17.81 \\
\hline $\mathbf{3 0 8}$ & 8.20 & 16.05 & 14.99 & 26.53 & 29.55 & 33.34 & 35.05 & 33.88 & 31.90 & 25.71 & 23.83 & 20.13 \\
\hline $\mathbf{3 1 3}$ & 8.20 & 16.05 & 14.99 & 30.64 & 32.08 & 33.34 & 38.63 & 39.64 & 37.07 & 36.92 & 29.75 & 31.35 \\
\hline $\mathbf{3 1 8}$ & 8.20 & 16.05 & 18.44 & 30.64 & 33.64 & 35.99 & 40.14 & 39.64 & 41.78 & 41.97 & 38.42 & 41.05 \\
\hline $\mathbf{3 2 3}$ & 8.20 & 16.05 & 18.44 & & 33.64 & 35.99 & 40.14 & 39.64 & & 45.52 & 44.82 & \\
\hline $\mathbf{3 2 8}$ & 10.60 & 16.05 & 18.44 & 30.64 & 33.64 & 35.99 & 40.14 & 39.64 & 41.24 & 46.24 & 44.74 & 47.34 \\
\hline $\mathbf{3 3 3}$ & 10.60 & 19.81 & 18.44 & 30.64 & 33.64 & 35.99 & 45.90 & 39.64 & 41.24 & 47.59 & 45.87 & 48.55 \\
\hline $\mathbf{3 3 8}$ & 10.60 & 25.47 & 32.31 & 33.66 & 33.64 & 40.67 & 45.90 & 39.64 & 45.43 & 49.78 & 48.00 & 49.56 \\
\hline $\mathbf{3 4 3}$ & 13.73 & 25.47 & 32.31 & 33.66 & 39.09 & 47.04 & 45.90 & 48.13 & 51.61 & 49.78 & 55.43 & 49.56 \\
\hline
\end{tabular}

Fig. 4 Averaged intensity of the bands B1-B5 (top) and BA-BB (below) obtained from the adjustment of the spectra of $\mathrm{Na}_{2}\left(5^{\prime}-\mathrm{GMP}\right)$ in Fig. 2. Since these bands are associated with G4 and stack conformations, the intensity of yellow and blue colour, respectively, represents the concentrations and temperatures where these conformations are more common.

The bands of the three oligomers studied at high temperatures after denaturation (Table S1, ESI $\dagger$ ) are roughly the same and coincide with those of the single-stranded DNA model measured previously. ${ }^{20}$

\subsection{Vibrational normal-mode calculations}

In order to provide an atomistic-level insight into our experiments, the Raman-active modes of several small G-quadruplex structures (2- or 3-layers) built with guanine and metallic cations $\left(\mathrm{Na}^{+}\right.$or $\left.\mathrm{K}^{+}\right)$were calculated using DFT (see Note 3, ESI $\dagger$ ). Regardless of the configuration of the structures, the vibrational modes obtained in the region of interest could be classified into three categories: interlayer displacements, which occurred at frequencies below $1 \mathrm{THz}$; collective hydrogen bond stretching and bending vibrations, in the range between 1-4.5 THz; and intramolecular vibrational modes of the guanine molecules, with frequencies over $4.5 \mathrm{THz}$. The modes belonging to the first two categories are purely intermolecular and are the most intense in the low-frequency region of the simulated OKE spectra (Fig. S19S21, ESI $\dagger$ ), especially in the complexes formed with potassium cations. 


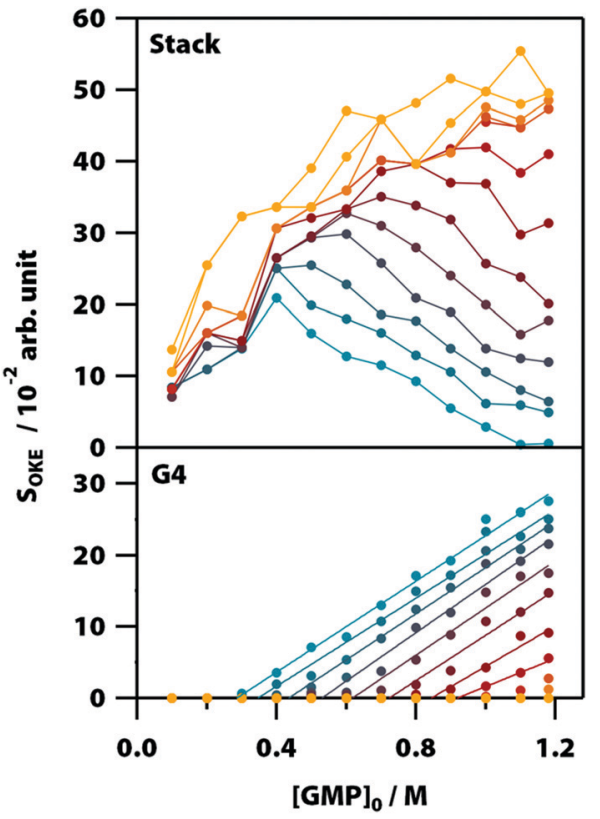

Fig. 5 Influence of temperature and initial concentration of GMP on the averaged OKE intensity of the bands associated with the stack (top) and G-quadruplex (bottom) conformations showing the delayed onset of the latter. Temperatures run between 283 (blue) to $318 \mathrm{~K}$ (red) in steps of $5 \mathrm{~K}$ and from $318 \mathrm{~K}$ to 358 (orange) in steps of $10 \mathrm{~K}$.

\subsection{Summary of results}

Here we have shown that the terahertz-frequency vibrational spectra of $\mathrm{Na}_{2}\left(5^{\prime}\right.$-GMP) in aqueous solution are generated by the combination of the base spectra from two different structures whose proportion depends on their concentration and temperature. It was demonstrated that the predominant base spectrum at high temperature or low concentration is due to the vibrational modes associated with the stacked nucleotide structure. This base spectrum, which is the only one observed in solutions of other nucleotides that only stack, could be modelled using two slightly underdamped Brownian oscillators.

It was demonstrated that the second base spectrum is due to vibrations associated with the G-quadruplex (G4) conformation and could be observed in G4s consisting of GMP nucleotides as well as three exemplar G4-forming oligonucleotides. This demonstrates that this base spectrum is largely characteristic of the G4 structure and not very much dependent on the exact base sequence. These associations between spectra and structures allow one to determine the stability of G4s from the ratio between the intensities of both base spectra and to determine the effect that factors such as temperature or the size of the counterion have on that stability. Thus, it was confirmed that the observed structures behaved as expected, except in the experiments with GMP and $\mathrm{Li}^{+}$, whose spectra indicated that this cation does not destabilize the G4 structures, as suggested recently. ${ }^{30}$

The G4-associated base spectrum can be fitted using six underdamped oscillator functions with centre frequencies in the 0.4-5.5 THz range. Apart from the lowest-frequency band B1, the observed modes have a similar damping rate of around $0.5 \mathrm{THz}$. Therefore, the viscous drag of the hydration shell that dissipates the energy stored in the oscillations is less efficient as the frequency of the bands increases, enabling coherent vibrations within the nucleic acid structure.

The experiments presented here provide more information about the vibrational modes detected in G4s. The band B1 is highly unusual with a peak frequency of only $\sim 400 \mathrm{GHz}$ and a damping rate of 150-190 GHz making it highly underdamped. In all liquids, solutions, and biomolecules in aqueous solution studied in this frequency region, an underdamped mode below $1 \mathrm{THz}$ has never been observed except in vitrified solutions ${ }^{31,32}$ and crystals. ${ }^{33,34}$ According to our vibrational normal-mode calculations, this band could correspond to the vibrational mode in which the layers of G-quartets are displaced perpendicular to the quartet plane. This interpretation is supported by the shift to lower frequency exhibited by this band when the inner cation is $\mathrm{Cs}^{+}$. As shown by structures characterized by X-ray crystallography (PDB entries: 2GW0, 1K8P and 1JB7), cations larger than potassium distort the guanine quartets, increasing the space between layers. The band B2 peaks at approximately double the frequency of the B1 band and has the same behaviour towards the cations of the inner channel, suggesting that this might be an overtone. The fact that in our simulated spectra there are no other modes with notable Raman activities in the sub-1 $\mathrm{THz}$ region supports this assignment (the calculations were performed with a double harmonic approximation and only fundamentals were considered when simulating the spectra).

The properties of the band B3 in the solutions of GMP are independent of the coordinating cation. However, the band is shifted to a higher frequency in the studied oligomers. Therefore, this mode is influenced by the restriction in the movement caused by the bonding between monomers. The fact that bands B3 (in G4-forming GMP and oligonucleotides), BE (in melted G4-forming oligonucleotides), and BE (in other DNA oligonucleotides, either single-stranded or double-stranded) all have similar vibrational parameters at the same frequency implies that the bases of the nucleotides do not participate in this vibrational mode, but are due to the interactions between the sugar and phosphate groups.

The band B4 is an underdamped vibrational mode that shifts to a higher frequency when potassium, the cation that most stabilizes the structure, is present. The band $\mathrm{B} 4$ in the

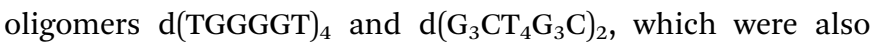
measured in solutions with $\mathrm{KCl}$, have characteristics almost identical to the spectra of the GMP. $\mathrm{K}^{+}$complex. This dependence of $\mathrm{K}^{+}$ excludes that, although they appear in the same frequency and have a similar damping ratio, the $\mathrm{B} 4$ band in the oligomers and the $\mathrm{BF}$ band observed in the double helix of the DNA are related.

The bands B5 and B6 are two highly underdamped vibrational modes that appear exclusively in the OKE spectra of nucleic acids in the G4 conformation. Their parameters are roughly equal in all the compounds studied here.

Our calculations suggest that while the B6 band is due to intramolecular vibrations, the B3, B4, and B5 bands correspond to vibrational modes in which the hydrogen bonds between the guanine bases are stretched and compressed. For B3, the normal mode peaking at approximately $2 \mathrm{THz}$ is indeed present in all our calculations showing a very minor dependence on the 


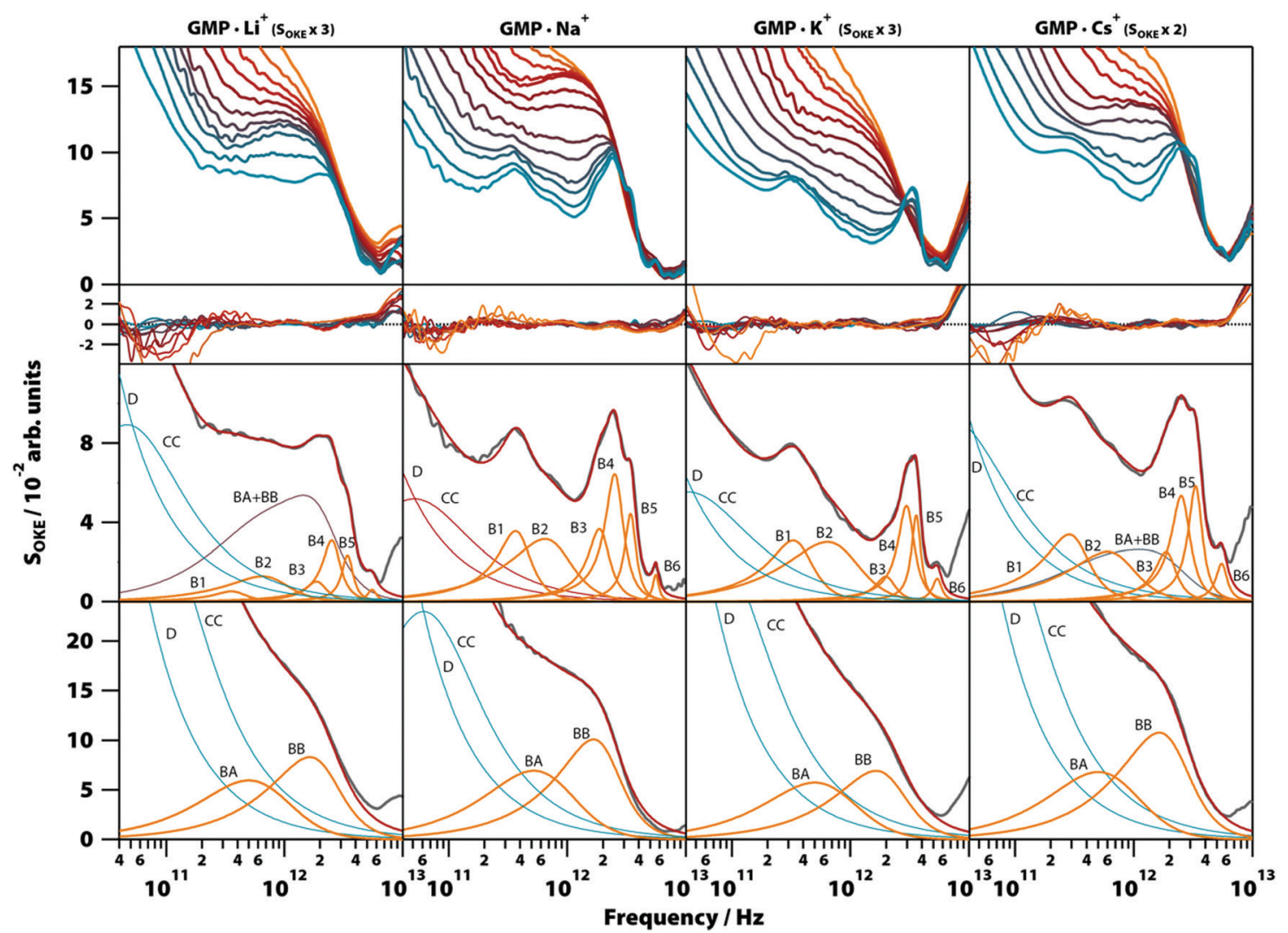

Fig. 6 Temperature-dependent OKE spectra of GMP solutions with different cations. Top row, from left to right: OKE spectra of GMP in the presence of Li ${ }^{+}$ (GMP 0.25 M, LiCl 1.0 M), $\mathrm{Na}^{+}$( $\left.\mathrm{Na}_{2}\left(5^{\prime}-\mathrm{GMP}\right) 1.0 \mathrm{M}\right), \mathrm{K}^{+}$(GMP 0.2 M, KCl $0.5 \mathrm{M}$ ), and $\mathrm{Cs}^{+}$solutions (GMP $0.4 \mathrm{M}, \mathrm{CsCl} 1.0$ M). The temperature runs from 283 (blue) to $328 \mathrm{~K}$ (red) in steps of $5 \mathrm{~K}$ and from $328 \mathrm{~K}$ to 358 (orange) in steps of $10 \mathrm{~K}$. Below the residuals between each spectrum and the fittings are shown. The remaining rows contain the spectrum for each case (grey) at $283 \mathrm{~K}$ (third row) and $358 \mathrm{~K}$ (fourth row) and the functions employed to fit the spectra. Diffusion associated functions (Debye and Cole-Cole) are coloured in red and vibration associated functions (Brownian oscillators) in orange. The blue band in the GMP. $\mathrm{Li}^{+}$and GMP.Cs${ }^{+}$spectra at $283 \mathrm{~K}$ is the result of the sum of the two Brownian oscillators associated with GMP stack conformation (BA + BB).

size of the stack and the cation. Moreover, it seems to be typical for this kind of vibration to have a notable Raman activity for different pairs of nucleobases (see Note 3, ESI $\dagger$ ). Thus, one could argue that B3 is not necessarily attributed to the interactions between the sugar and phosphate groups. From this regard, motions that could be account for B4 and B5 bands seem to be more exclusive to G4-related structures.

Fig. 5 shows the influence of the initial concentration of GMP on the intensity of each base spectrum for each temperature. These results show that the measured GMP solutions follow the chemical equilibria shown in Fig. 1c. As the concentration increases, the signal of the GMP stacks increases until the formation of G4 structures is favoured and begins to descend. In the case of the signal associated with G-quadruplexes, it rises linearly, as much as the temperature allows, as the GMP concentration increases.

\section{Discussion and conclusions}

The low-frequency dynamics of DNA can be modelled theoretically using simple ball and spring models, ${ }^{35}$ coarse-grained models, ${ }^{36}$ and atomistic molecular models ${ }^{37}$ that incorporate the hydrogen-bond bends and stretches of paired bases as well as other low-frequency motions in the DNA backbone. Such models can predict certain traits of inelastic X-ray and neutron scattering data obtained from dry samples of DNA and RNA. $^{38,39}$ Coarse-grained models analysed using Instantaneous Normal Modes (INM) suggest that terahertz-frequency modes in DNA can be understood in terms of delocalized phonon-like modes in an approximately one-dimensional structure, localized by disorder and interactions with the surrounding solvent. ${ }^{40}$ In a perfect (ordered, undamped, and infinite) lattice, Raman scattering (including OKE) should only occur from optical phonons at the zone centre (that is with a wavelength delocalized over the entire structure). However, in the presence of disorder and damping, momentum selection rules are expected to break down and Raman-scattering will take place from essentially all optical and acoustic phonon modes. Previous simulations ${ }^{40}$ have suggested that this should lead to a broad 2-3 THz band but that is not seen in these experiments with dsDNA and G4 structures. Similarly, a study on highly hydrated DNA fibres, combining high-resolution inelastic x-ray scattering experiments and incoherent inelastic neutron scattering, shows that two different collective modes contribute to the low-frequency dynamics of DNA: a localized optical-like mode at $\sim 0.5 \mathrm{THz}$ and an 


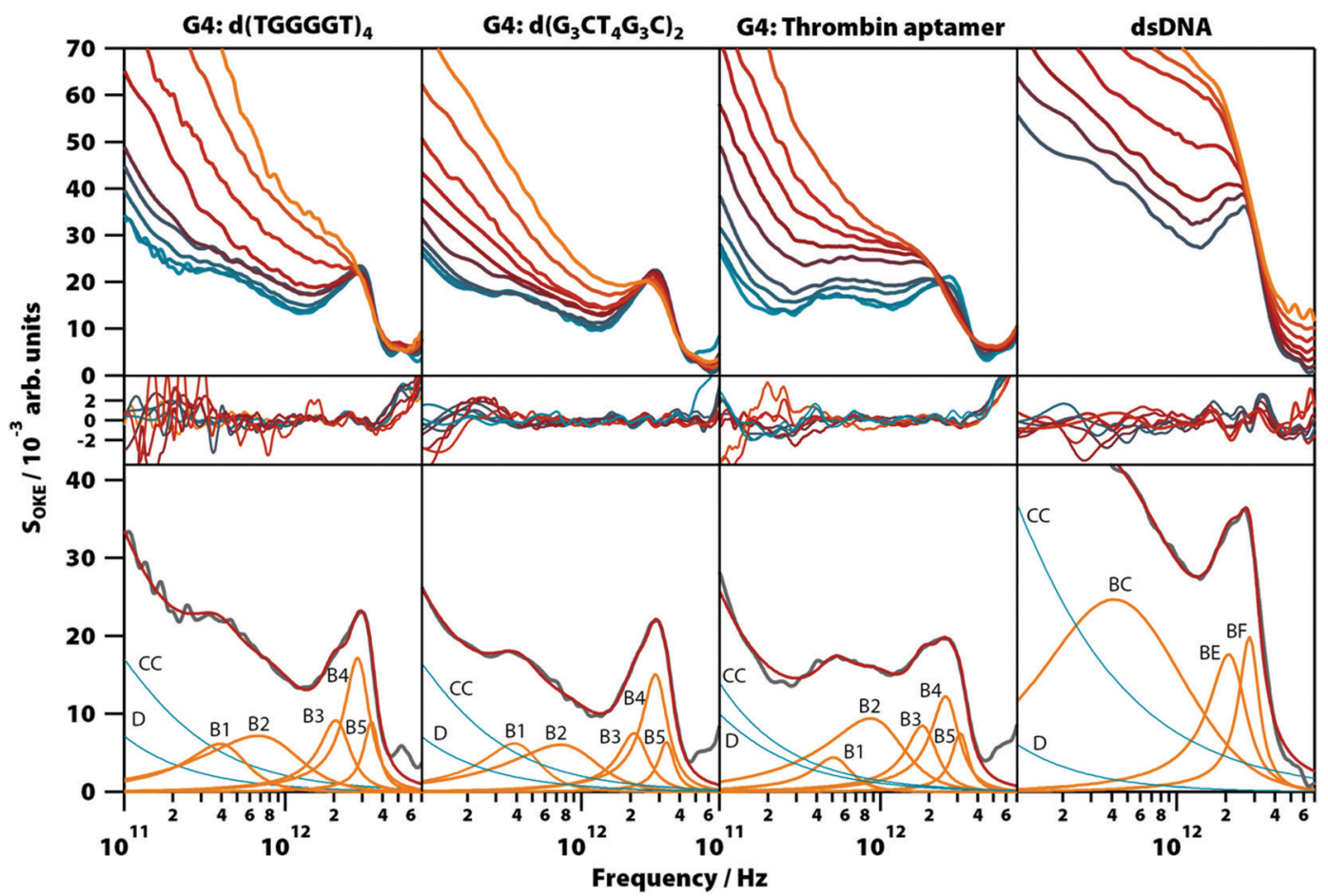

Fig. 7 OKE spectra of solutions of three G4-forming oligomers and one double-helix forming oligomer demonstrating that the gigahertz-to-terahertz modes seen in GMP-based G-quadruplexes persist in oligomers. From left to right: Spectra of d(TGGGGT) $\left(80 \mathrm{mg} \mathrm{ml}^{-1}, \mathrm{KCl}_{1.6 ~} \mathrm{M}\right), \mathrm{d}\left(\mathrm{G}_{3} \mathrm{CT}_{4} \mathrm{G}_{3} \mathrm{C}\right)$ $\left(80 \mathrm{mg} \mathrm{ml}^{-1}, \mathrm{KCl} 1.6 \mathrm{M}\right.$ ), and TBA ( $80 \mathrm{mg} \mathrm{ml}^{-1}, \mathrm{KCl} 0.4 \mathrm{M}$ ) between 283 (blue) to $318 \mathrm{~K}$ (red) in steps of $5 \mathrm{~K}$ and from $318 \mathrm{~K}$ to 358 (orange) in steps of $10 \mathrm{~K}$. Spectra of $d\left(T_{2} A_{2} A_{2} A_{3} T T_{A} T_{3} A_{2} T A_{2}\right)_{2}$ (right) between 298 (blue) and $358 \mathrm{~K}$ (orange) in intervals of $10 \mathrm{~K}$. Residuals are shown below. The third row shows the spectrum of lower temperature for each oligomer (grey) and the functions used to model them. Debye and Cole-Cole functions are coloured in red and Brownian oscillators in orange.

acoustic-like mode in the region of 3-5 THz, characterized by large damping, which propagates along the DNA fibre axis. ${ }^{41}$ In addition, normal-mode analysis applied to different nucleic acids $^{38,42-44}$ results in a relatively flat distribution of lowfrequency modes from 0 to $5-8 \mathrm{THz}\left(150-250 \mathrm{~cm}^{-1}\right)$ in which the modes with frequencies $<1 \mathrm{THz}$ have the greatest contribution to the motion of the atoms of the molecule. This is inconsistent with the data shown here: the spectra consist of a small number (2-5) of homogeneously broadened bands corresponding to a small number of underdamped modes. Although it is true that the homogeneously broadened nature of modes hidden in the middle of the spectra is difficult to ascertain, the spectra show distinct peaks (more readily observable on a linear frequency axis as shown in, for example, Fig. S1, ESI $\dagger$ ) proving the presence of underdamped modes. This is quite unlike the broad range of finely spaced normal modes predicted by theoretical models. Thus, current vibrational models of DNA fail to predict the small number of distinct vibrational bands observed in this work.

It is usually assumed that terahertz frequency modes in molecules in aqueous solution will be strongly overdamped. ${ }^{41,45}$ However, the data presented here show that this is not the case: double-stranded DNA has modes at $\sim 2 \mathrm{THz}$ that are underdamped (damping rate $0.5-0.7 \mathrm{THz}$ ) while the G4 structure has modes at even higher frequencies (approximately 2, 2.6, 3.4, and $5.5 \mathrm{THz}$ ). As can be seen in Table S1 (ESI + ), the damping of the modes labelled BA and BB in nucleotide stacks is approximately $1 \mathrm{THz}$, whereas that of the modes labelled B1-B6 in the G4 structures ranges from 0.15 to $0.5 \mathrm{THz}$. This reduced damping reflects the greater rigidity of the G4 structure over dsDNA and reduced coupling to the solvent. As $k_{\mathrm{B}} T / h$ at room temperature is $6.1 \mathrm{THz}$, it is these modes that play the most important role in determining the vibrational enthalpy and entropy.

Long-distance signal transmission and allostery have been demonstrated in DNA samples when the biomolecule interacts with a ligand, e.g., in anticancer drugs, ${ }^{46}$ small molecules ${ }^{47}$ or binding proteins. ${ }^{48}$ Mechanisms have been proposed in which the ligand distorts the double helix, increasing the affinity of the second ligand. ${ }^{47}$ However, it has been shown that allostery in DNA when it binds to a eukaryotic transcription factor and a type II endonuclease occurs without changing its conformation. $^{48}$ The mechanism of allostery without conformational change has been suggested to involve thermally excited low-frequency vibrational modes travelling long distances in the nucleic acid. This hypothesis is compatible with the oscillatory distance dependence of the allosteric interaction mediated by DNA found by several models. ${ }^{48-50}$ This allostery is largely dependent on the helical structure of the linker DNA and the changes in its mechanical properties: as the interaction with the first DNA-binding protein stiffens the DNA, the entropic penalty for binding the second DNA protein is less resulting in allostery. 
Our results show that a change in the stiffness of nucleic acids modifies their vibrational profile. Nucleotide G4s have phonon bands at a higher frequency than in dsDNA that also have smaller damping rates, so the coherent oscillations decay more slowly. These changes will result in a free energy penalty that is compensated for by the interaction with the central cation and hence binding to these structures will incur a muchreduced entropic penalty. This effect was also observed in G4 structures formed by GMP when the inner cation was changed. The structures most stabilized by the inner cation, and therefore the stiffest, are the structures that show phonon bands at a higher frequency. Likewise, the bands observed in the G4 structures with their nucleotides linked by phosphodiester bonds, d(TGGGGT $)_{4}$, TBA, and $\mathrm{d}_{(}\left(\mathrm{G}_{3} \mathrm{CT}_{4} \mathrm{G}_{3} \mathrm{C}\right)_{2}$, appear at a higher frequency than the bands of $\mathrm{G} 4$ structures formed by free GMP molecules. All these experiments will be useful to test the role that phonon-like modes play in allosteric interaction and, as a result, in gene regulation. ${ }^{51}$

\section{Conflicts of interest}

There are no conflicts to declare.

\section{Acknowledgements}

We thank the Engineering and Physical Sciences Research Council (EPSRC) for support through grants EP/J009733/1, EP/ K034995/1, EP/N508792/1, and EP/N007417/1. This work was partially funded by Leverhulme Trust Research Project Grant RPG-2018-350 and received funding from the European Research Council (ERC) under the European Union's Horizon 2020 research and innovation program (grant agreement No. 832703).

\section{References}

1 G. Biffi, D. Tannahill, J. McCafferty and S. Balasubramanian, Quantitative visualization of DNA G-quadruplex structures in human cells, Nat. Chem., 2013, 5, 182-186.

2 V. S. Chambers, G. Marsico, J. M. Boutell, M. Di Antonio, G. P. Smith and S. Balasubramanian, High-throughput sequencing of DNA G-quadruplex structures in the human genome, Nat. Biotechnol., 2015, 33, 877-881.

3 G. Laughlan, A. I. H. Murchie, D. G. Norman, M. H. Moore, P. C. E. Moody, D. M. J. Lilley and B. Luisi, The highresolution crystal structure of a parallel-stranded guanine tetraplex, Science, 1994, 265, 520-524.

4 T. J. Pinnavaia, H. T. Miles and E. D. Becker, Self-Assembled 5'-Guanosine Monophosphate. Nuclear Magnetic Resonance Evidence for a Regular, Ordered Structure and Slow Chemical Exchange, J. Am. Chem. Soc., 1975, 97, 7198-7200.

5 J. T. Davis, G-Quartets 40 Years Later: From 5'-GMP to Molecular Biology and Supramolecular Chemistry, Angew. Chem., Int. Ed., 2004, 43, 668-698.

6 D. Sen and W. Gilbert, Nature, 1990, 344, 410-414.
7 J. A. Capra, K. Paeschke, M. Singh and V. A. Zakian, G-quadruplex DNA sequences are evolutionarily conserved and associated with distinct genomic features in Saccharomyces cerevisiae, PLoS Comput. Biol., 2010, 6, 9.

8 R. H. Shafer and I. Smirnov, Biological aspects of DNA/RNA quadruplexes, Biopolymers, 2000, 56, 209-227.

9 A. Berroyer and N. Kim, The Functional Consequences of Eukaryotic Topoisomerase 1 Interaction with G-Quadruplex DNA, Genes, 2020, 11, 193.

10 H. Sun, J. K. Karow, I. D. Hickson and N. Maizels, The Bloom's syndrome helicase unwinds G4 DNA, J. Biol. Chem., 1998, 273, 27587-27592.

11 A. M. Zahler, J. R. Williamson, T. R. Cech and D. M. Prescott, Inhibition of telomerase by G-quartet DMA structures, Nature, 1991, 350, 718-720.

12 R. Navarro, F. Peral, E. Gallego and J. Morcillo, Studies of the self-association of $5^{\prime}$-GMP in aqueous solutions by Fourier transform infrared spectroscopy, J. Mol. Struct., 1986, 143, 357-360.

13 R. D. Gray, J. O. Trent and J. B. Chaires, Folding and unfolding pathways of the human telomeric G-quadruplex, J. Mol. Biol., 2014, 426, 1629-1650.

14 J. L. Mergny, A. De Cian, A. Ghelab, B. Saccà and L. Lacroix, Kinetics of tetramolecular quadruplexes, Nucleic Acids Res., 2005, 33, 81-94.

15 C. Kang, X. Zhang, R. Ratliff, R. Moyzis and A. Rich, Crystal structure of four-stranded Oxytricha telomeric DNA, Nature, 1992, 356, 126-131.

16 M. Meier, A. Moya-Torres, N. J. Krahn, M. D. McDougall, G. L. Orriss, E. K. S. McRae, E. P. Booy, K. McEleney, T. R. Patel, S. A. McKenna and J. Stetefeld, Structure and hydrodynamics of a DNA G-quadruplex with a cytosine bulge, Nucleic Acids Res., 2018, 46, 5319-5331.

17 A. Wong, R. Ida, L. Spindler and G. Wu, Disodium guanosine 5 -monophosphate self-associates into nanoscale cylinders at pH 8: A combined diffusion NMR spectroscopy and dynamic light scattering study, J. Am. Chem. Soc., 2005, 127, 6990-6998.

18 R. W. Harkness and A. K. Mittermaier, G-quadruplex dynamics, Biochim. Biophys. Acta, Proteins Proteomics, 2017, 1865, 1544-1554.

19 C. Phelps, W. Lee, D. Jose, P. H. von Hippel and A. H. Marcus, Single-molecule FRET and linear dichroism studies of DNA breathing and helicase binding at replication fork junctions, Proc. Natl. Acad. Sci. U. S. A., 2013, 110, 17320-17325.

20 M. González-Jiménez, G. Ramakrishnan, T. Harwood, A. J. Lapthorn, S. M. Kelly, E. Ellis and K. Wynne, Observation of coherent delocalised phonon-like modes in DNA under physiological conditions, Nat. Commun., 2016, 7, 11799.

21 G. Hithell, M. González-Jiménez, G. M. Greetham, P. M. Donaldson, M. Towrie, A. W. Parker, G. A. Burley, K. Wynne and N. T. Hunt, Ultrafast 2D-IR and optical Kerr effect spectroscopy reveal the impact of duplex melting on the structural dynamics of DNA, Phys. Chem. Chem. Phys., 2017, 19, 10333-10342.

22 G. Ramakrishnan, M. González-Jiménez, A. J. Lapthorn and K. Wynne, The Spectrum of Slow and Super-Slow (Picosecond 
to Nanosecond) Water Dynamics around Organic and Biological Solutes, J. Phys. Chem. Lett., 2017, 8, 2964-2970.

23 R. Rymdén and P. Stilbs, Nucleotide aggregation in aqueous solution. A multicomponent self-diffusion study, Biophys. Chem., 1985, 21, 145-156.

24 P. Schultze, N. V. Hud, F. W. Smith and J. Feigon, The effect of sodium, potassium and ammonium ions on the conformation of the dimeric quadruplex formed by the Oxytricha nova telomere repeat oligonucleotide d(G4T4G4), Nucleic Acids Res., 1999, 27, 3018-3028.

25 M. J. Frisch, G. W. Trucks, H. B. Schlegel, G. E. Scuseria, M. A. Robb, J. R. Cheeseman, G. Scalmani, V. Barone, G. A. Petersson, H. Nakatsuji, X. Li, M. Caricato, A. Marenich, J. Bloino, B. G. Janesko, R. Gomperts, B. Mennucci, H. P. Hratchian, J. V. Ortiz, A. F. Izmaylov, J. L. Sonnenberg, D. Williams-Young, F. Ding, F. Lipparini, F. Egidi, J. Goings, B. Peng, A. Petrone, T. Henderson, V. G. Ranasinghe, D. Zakrzewski, J. Gao, N. Rega, G. Zheng, W. Liang, M. Hada, M. Ehara, K. Toyota, R. Fukuda, J. Hasegawa, M. Ishida, T. Nakajima, Y. Honda, O. Kitao, H. Nakai, T. Vreven, K. Throssell, J. A. Montgomery, J. E. Peralta, F. Ogliaro, M. Bearpark, J. J. Heyd, E. Brothers, K. N. Kudin, V. N. Staroverov, T. Keith, R. Kobayashi, J. Normand, K. Raghavachari, A. Rendell, J. C. Burant, S. S. Iyengar, J. Tomasi, M. Cossi, J. M. Millam, M. Klene, C. Adamo, R. Cammi, J. W. Ochterski, R. L. Martin, K. Morokuma, O. Farkas, J. B. Foresman and D. J. Fox, Gaussian 09, Gaussian, Inc., Wallingford, CT, 2013.

26 S. Grimme, S. Ehrlich and L. Goerigk, Effect of the damping function in dispersion corrected density functional theory, J. Comput. Chem., 2011, 32, 1456-1465.

27 T. J. Pinnavaia, C. L. Marshall, C. L. Fisk, H. T. Miles and E. D. Becker, Alkali Metal Ion Specificity in the Solution Ordering of a Nucleotide, 5'-Guanosine Monophosphate, J. Am. Chem. Soc., 1978, 100, 3625-3627.

28 W. Eimer and T. Dorfmuller, Self-Aggregation of Guanosine 5'-Monophosphate Studied By Dynamic Light-Scattering Techniques, J. Phys. Chem., 1992, 96, 6790-6800.

29 E. A. Venczel and D. Sen, Parallel and Antiparallel G-DNA Structures from a Complex Telomeric Sequence, Biochemistry, 1993, 32, 6220-6228.

30 D. Bhattacharyya, G. Mirihana Arachchilage and S. Basu, Metal Cations in G-Quadruplex Folding and Stability, Front. Chem., 2016, 4, 1-14.

31 D. A. Turton, J. Hunger, A. Stoppa, A. Thoman, M. Candelaresi, G. Hefter, M. Walther, R. Buchner and K. Wynne, Rattling the cage: Micro- to mesoscopic structure in liquids as simple as argon and as complicated as water, J. Mol. Liq., 2011, 159, 2-8.

32 J. Reichenbach, S. A. Ruddell, M. González-Jiménez, J. Lemes, D. A. Turton, D. J. France and K. Wynne, Phonon-like Hydrogen-Bond Modes in Protic Ionic Liquids, J. Am. Chem. Soc., 2017, 139, 7160-7163.

33 K. A. Niessen, M. Xu, D. K. George, M. C. Chen, A. R. FerréD’Amaré, E. H. Snell, V. Cody, J. Pace, M. Schmidt and A. G. Markelz, Protein and RNA dynamical fingerprinting, Nat. Commun., 2019, 10, 1-10.
34 K. A. Niessen, M. Xu, A. Paciaroni, A. Orecchini, E. H. Snell and A. G. Markelz, Moving in the Right Direction: Protein Vibrational Steering Function, Biophys. J., 2017, 112, 933-942.

35 K. C. Chou, Low-frequency vibrations of DNA molecules, Biochem. J., 1984, 221, 27-31.

36 T. A. Knotts, N. Rathore, D. C. Schwartz and J. J. De Pablo, A coarse grain model for DNA, J. Chem. Phys., 2007, 126, 084901.

37 F. Merzel, F. Fontaine-Vive, M. Johnson and G. Kearley, Atomistic model of DNA: Phonons and base-pair opening, Phys. Rev. E: Stat., Nonlinear, Soft Matter Phys., 2007, 76, 031917.

38 D. Lin, A. Matsumoto and N. Go, Normal mode analysis of a double-stranded DNA dodecamer d(CGCGAATTCGCG), J. Chem. Phys., 1997, 107, 3684-3690.

39 T. E. Cheatham and D. A. Case, Twenty-five years of nucleic acid simulations, Biopolymers, 2013, 99, 969-977.

40 S. Cocco and R. Monasson, Theoretical study of collective modes in DNA at ambient temperature, J. Chem. Phys., 2000, 112, 10017-10033.

41 A. Paciaroni, L. Comez, M. Longo, F. Sebastiani, F. Bianchi, A. Orecchini, M. Zanatta, R. Verbeni, A. Bosak, F. Sacchetti and C. Petrillo, Terahertz collective dynamics of DNA as affected by hydration and counterions, J. Mol. Liq., 2020, 318, 113956.

42 T. Duong and K. Zakrzewska, Calculation and analysis of low frequency normal modes for DNA, J. Comput. Chem., 1997, 18, 796-811.

43 M. Bykhovskaia, B. Gelmont, T. Globus, D. L. Woolard, A. C. Samuels, T. H. Duong and K. Zakrzewska, Prediction of DNA far-IR absorption spectra based on normal mode analysis, Theor. Chem. Acc., 2001, 106, 22-27.

44 A. E. Garcia and D. M. Soumpasis, Harmonic vibrations and thermodynamic stability of a DNA oligomer in monovalent salt solution, Proc. Natl. Acad. Sci. U. S. A., 1989, 86, 3160-3164.

45 M. D. Frank-Kamenetskii and S. Prakash, DNA theoretical modeling Reply to comments on 'Fluctuations in the DNA double helix: A critical review', Phys. Life Rev., 2014, 11, 181-183.

46 T. R. Krugh and M. A. Young, Daunorubicin and adriamycin facilitate actinomycin D binding to poly(dA-dT).poly(dA-dT), Nature, 1977, 269, 627-628.

47 D. M. Chenoweth and P. B. Dervan, Allosteric modulation of DNA by small molecules, Proc. Natl. Acad. Sci. U. S. A., 2009, 106, 13175-13179.

48 S. Kim, E. Brostromer, D. Xing, J. Jin, S. Chong, H. Ge, S. Wang, C. Gu, L. Yang, Y. Q. Gao, X.-D. Su, Y. Sun and X. S. Xie, Probing Allostery Through DNA, Science, 2013, 339, 816-819.

49 A. Balaceanu, A. Pérez, P. D. Dans and M. Orozco, Allosterism and signal transfer in DNA, Nucleic Acids Res., 2018, 46, 7554-7565.

$50 \mathrm{~J}$. Singh and P. K. Purohit, Elasticity as the Basis of Allostery in DNA, J. Phys. Chem. B, 2019, 123, 21-28.

51 M. M. Saha and T. C. Kofane, Long-range interactions between adjacent and distant bases in a DNA and their impact on the ribonucleic acid polymerase-DNA dynamics, Chaos, 2012, 22, 013116. 